\title{
NMR relaxation parameters from molecular simulations of hydrated inorganic nanopores
}

\author{
Jayesh S. Bhatt*† Peter J. McDonald*, David A. Faux*, \\ Nicholas C. Howlett*, Sergey V. Churakov ${ }^{\ddagger}$
}

\begin{abstract}
Nuclear magnetic resonance (NMR) relaxometry is a powerful technique to characterise diffusive motion of fluids in nanoporous to microporous media. Molecular simulations can be used to predict NMR relaxation parameters using a dipolar spin-spin correlation function. In this Paper, molecular dynamics simulations of water diffusion in anomalous $11 \AA$ tobermorite, consisting of three slit pores and one gel pore of width $\sim 1.0 \mathrm{~nm}$, have been performed. The spin-spin correlation function components corresponding to both $2 \mathrm{D}$ and quasi-2D translation and rotation of water are presented. It was found that motion in the slit pores is highly correlated, leading to a significantly shorter relaxation time compared to bulk water. The correlation between the slit pores and the gel pore was found to be negligible compared to that within either the gel pore or the slit pore exclusively. Nevertheless, this correlation function can be useful in quantifying water diffusion within the slit pores, which occurs primarily through stochastic site jumping. It was found that stronger surface interaction leads to lower relaxation times, while the hydroxyls on the surface help further lower the water relaxation times.
\end{abstract}

${ }^{*}$ Department of Physics, University of Surrey, Guildford, GU2 7XH, UK

†bhatt@physics.org

${ }_{\ddagger}^{\ddagger}$ Paul Scherrer Institute, Laboratory for Waste Management, CH-5232 Villigen-PSI, Switzerland 


\section{INTRODUCTION}

Diffusion in porous media has received considerable attention in recent years due to its relevance to problems as diverse as heterogeneous catalysis, oil recovery from rocks, food processing and cement science. Systems in which the diffusing fluid is protonated can be studied using nuclear magnetic resonance (NMR) relaxation analysis experiments of the fluid owing to the nuclear magnetic moment of hydrogen ${ }^{1,2}$. Water and oil are two prime examples of such fluids. Experimentally, it is well known that the NMR relaxation rate of fluids in small pores increases as the pore volume to surface ratio, or size, decreases. However, while NMR relaxometry experiments are excellent for probing the surface to volume ratio of the pore space confining the fluid in nanoscopic to microscopic porous media ${ }^{3}$, one must still resort to computational modelling ${ }^{4,5}$ in order to understand the fluid movement at the nanoscopic scale and the chemical topology of the internal surfaces of the porous media. Recent advances in computational power, as well as advances in high frequency experiment, mean that simulations of molecular trajectories of sufficient length can be made to enable the calculation of relaxation rates at (increasingly low) frequencies that overlap with (increasingly high) frequency experiment. Results from the simulations may therefore be compared to those from experiments.

A theoretical framework for calculating NMR relaxation rates in an idealised fluid of non-interacting spins, known as the BPP theory ${ }^{6}$, was formulated by Bloembergen, Purcell and Pound. NMR relaxation rates were first calculated using Monte Carlo (MC) simulations of spins hopping on a lattice in $1986^{7}$, wherein earlier theoretical work of Sholl on dipolar spin-spin correlation functions was utilised ${ }^{8,9}$. Grivet obtained relaxation parameters from a molecular dynamics (MD) simulation of a Lennard-Jones fluid of hypothetical particles and discussed issues that may arise while performing such calculations on water ${ }^{10}$. Calculation of NMR relaxation rates using MD simulations have also been done with considerable success in the context of biologically relevant systems ${ }^{11-14}$. However, most studies involving biological molecules focus primarily on the molecular reorientational dynamics. Faux et al. recently used MD and MC simulations to obtain results of NMR relaxation parameters arising from water diffusion between two slabs of $\alpha$-quartz $\left(\mathrm{SiO}_{2}\right)$ that included a detailed mathematical 
framework capturing the angular as well as the relative translational motion of spins over a long range. ${ }^{15}$ This model was successful in calculating a bulk water spin-lattice relaxation time of $4.3 \pm 0.4 \mathrm{~s}$ at $150 \mathrm{MHz}$ Larmor frequency and $300 \mathrm{~K}$, as compared to the experimental value of $3.8 \mathrm{~s}$.

This work is motivated by ongoing desire to characterise the nanoscale porosity and morphology of calcium-silicate-hydrates (C-S-H), the active phase of cement ${ }^{16,17}$, given the importance of cement as a construction material, an interest to which NMR is making an increasingly important contribution ${ }^{18}$. However, many of its broader conclusions are equally applicable to other nanoporous materials such as zeolites ${ }^{19,20}$ and layered double hydroxides $(\mathrm{LDH})^{21}$. C-S-H is a highly heterogeneous, nanoporous material comprising disordered sheets of calcium and oxygen atoms and silicate tetrahedra separated by sheets of water ${ }^{22}$. Regions of stacked sheets are reported to be separated by nano-sized gel pores and larger capillary pores for which different morphologies have been proposed ${ }^{23,24}$. Tobermorite is a naturally occurring mineral ${ }^{25}$ that is often considered as a reference structural model for C-S-H stacked sheets. Tobermorite has a layered structure represented by sheets of seven-fold coordinated calcium ions. Silica tetrahedra form wollastonite-like chains attached to the calcium layer. The interlayer space between sheets is occupied by water molecules and hydrated calcium ions. In the anomalous $11 \AA$ phase of tobermorite there is no calcium in the interlayer and hydroxyl groups are formed on the interlayer surfaces. The detailed structural position of water and ions in the interlayer of anomalous $11 \AA$ tobermorite has been derived recently by $a b$ initio molecular dynamics simulations. ${ }^{26,27}$

In this Paper we use a slightly modified $a b$ initio tobermorite structure as an analogue of C-S-H to calculate NMR relaxation parameters of water in C-S-H based on classical MD simulations. The modification comprises of expanding one slit pore and filling it with additional water. By this means the nanoporosity of C-S-H with a bimodal distribution of largely planar pores, consisting of very narrow interlayer or slit-pore spaces and gel pores of the order of 1-5 nm wide, is reproduced.

The objectives of this work are to quantify water diffusion in slit pores and to explore the relative importance of two-dimensional (2D) slit pores and quasi-two-dimensional (Q2D) gel pores on the measured relaxation. While Faux et al. ${ }^{15}$ previously explored the effect of vary- 
ing the size of a single gel pore on relaxation times using quartz as a model system, there is no prior modelling work that examines the effect of having two pores of different size in close proximity. Moreover, to our knowledge, very little literature exists on the nature of water diffusion in slit pores of C-S-H and no information is available about the water translational correlation functions leading to experimentally observed ${ }^{1} \mathrm{H}$ NMR relaxation times in these pores. In this work, the chosen tobermorite system allows us to assess the combined effect of having 2D and Q2D pores in the same system. The motivation of the work is to establish the validity of assumptions incorporated into analytical models of relaxation in porous systems by exploring the fast-exchange assumption, determining the relative contributions of the rotational and translational motion of spins, measuring the surface residence time and exploring whether experimental relaxation times contain contributions due to water interaction with surface hydroxyls. Throughout the work, the different contributions to relaxation due to nuclei in different environments are separately calculated to explore their relative importance.

\section{METHODOLOGY}

\section{Theory}

An NMR relaxometry experiment measures the spin-lattice $\left(T_{1}\right)$ and spin-spin $\left(T_{2}\right)$ relaxation times in the presence of an external static magnetic field and these times can be expressed in terms of spectral density functions $J^{(m)}(\omega)$. For a system involving the diffusion of the same nuclear species, the NMR relaxation rates are related to the spectral densities as ${ }^{28}$

$$
\begin{gathered}
\frac{1}{T_{1}}=\frac{3}{2}\left(\frac{\mu_{0}}{4 \pi}\right)^{2} \gamma^{4} \hbar^{2} I(I+1)\left[J^{(1)}(\omega)+J^{(2)}(2 \omega)\right], \\
\frac{1}{T_{2}}=\frac{3}{8}\left(\frac{\mu_{0}}{4 \pi}\right)^{2} \gamma^{4} \hbar^{2} I(I+1)\left[J^{(0)}(0)+10 J^{(1)}(\omega)+J^{(2)}(2 \omega)\right],
\end{gathered}
$$

where $\gamma$ and $I$ are the gyromagnetic ratio and spin of the diffusing spins and $\omega$ is the Larmor frequency in the applied static field. The spectral density functions, $J^{(m)}(\omega)$, measure how much magnetic field fluctuation a given spin experiences due to the presence of other spins 
in its surroundings. These can be calculated from the Fourier transformation of the dipolar correlation functions, $G^{(m)}(t)$,

$$
\begin{aligned}
J^{(m)}(\omega) & =\int_{-\infty}^{\infty} G^{(m)}(t) e^{-i \omega t} d t \\
G^{(m)}(t) & =\alpha^{(m)}\left\langle\frac{Y_{2 m}\left(\theta_{0}, \phi_{0}\right) Y_{2 m}^{*}(\theta, \phi)}{r_{0}^{3} r^{3}}\right\rangle,
\end{aligned}
$$

where

$$
\alpha^{(0)}=\frac{48 \pi}{15}, \quad \alpha^{(1)}=\frac{8 \pi}{15}, \quad \alpha^{(2)}=\frac{32 \pi}{15} .
$$

Here, $Y_{2 m}(\theta, \phi)$ are the spherical harmonic functions involving relative position vectors between spin pairs that are $\mathbf{r}_{0}$ and $\mathbf{r}$ at $t=0$ and at time $t$ respectively. The spherical polar coordinates of the spin pairs are $\left(r_{0}, \theta_{0}, \phi_{0}\right)$ and $(r, \theta, \phi)$ repectively in the laboratory frame of reference that has the polar axis in the direction of the static magnetic field. The superscript on the spherical harmonic indicates the complex conjugate, while the ensemble average represented by Eq. (3) is the average over spins pairs.

Equation (3) holds true for a system held at a fixed orientation with respect to the external magnetic field. Real life experiments usually involve powdered or porous material in which pores are randomly oriented with respect to the magnetic field. An expression for the powder-averaged correlation function, $G^{*}(t)$, was obtained by Sholl ${ }^{8}$ in the case where the spins are diffusing in a three-dimensional (3D) manner, e.g. a simple three-dimensional box of fluid. On the other hand, two-dimensional (2D) diffusion occurs when a fluid is confined strictly to a flat 2D plane. In nanoporous materials, the fluid is often confined between planar membranes that are separated by a few nanometers. We refer to the confined fluid motion in such materials as quasi-2D. It was later shown by Faux et al. ${ }^{15}$ that the same expression for powder averaging is also valid for two-dimensional (2D) and quasi-two-dimensional (Q2D) diffusion of spins:

$$
\begin{aligned}
G^{*}(t) & =\frac{1}{N^{\prime}} \sum_{r_{0}, r>0} \frac{\frac{1}{2}\left(3 \cos ^{2} \psi-1\right)}{r_{0}^{3} r^{3}} \\
& =\left\langle\frac{P_{2}(\cos \psi)}{r_{0}^{3} r^{3}}\right\rangle
\end{aligned}
$$

where $N^{\prime}$ is the number of spins in the summation, $\psi$ is the angle between $\mathbf{r}$ and $\mathbf{r}_{\mathbf{0}}$ and $P_{2}(x)=\frac{1}{2}\left(3 \cos ^{2} x-1\right)$ is a Legendre polynomial. The angular brackets again represent the 
average over an ensemble of spin pairs. Furthermore, the powder-averaged $G^{*}(t)$ is related to $G^{(m)}(t)$ through

$$
G^{*}(t)=\frac{4 \pi G^{(m)}(t)}{\alpha^{(m)}} .
$$

The function $G^{*}(t)$ is independent of the value of $m$. Hence, given the Cartesian coordinates as a function of time from a molecular simulation, it is possible to work out the correlation function from Eq. (4) and the NMR relaxation times through Eqs. (1), (2) and (6).

In theory, there is a unique value of $T_{1}$ and $T_{2}$ attached to each spin in the system. This arises from the fact that a given spin interacts with every other spin in the system and the summation appearing in Eq. (4) reflects this. However, in practice the function $G^{*}(t)$ for an individual spin would generally exhibit extremely large statistical fluctuations. To smooth out these fluctuations, averaging over a large number of time origins would be required, making it a computationally expensive process. On the other hand, classifying spins according to their environments helps one understand the relative importance of each environment to the relaxation rates. This also has the added benefit that the $G^{*}(t)$ averaged over all the spins in the given class will have smaller fluctuations and obtaining a smooth $G^{*}(t)$ function will require a smaller number of time origins. It is therefore useful to identify which distinct magnetic environments exist and to what extent spin exchange between environments occurs. For instance, in a heterogeneous system one can classify all the spins as either bulk phase spins or surface phase spins. It is also important to identify the type of motion they are undergoing (e.g. translational, rotational, two-dimensional, three-dimensional). The relaxation time of a spin adsorbed on a surface would be much shorter (faster relaxation rate) than that of a bulk fluid spin.

If the spins appearing in the summation of Eq. (4) can be divided into different groups, then contributions due to each group to the relaxation rate can be evaluated. If all the spins have essentially the same dynamics, then a meaningful average can be calculated according to

$$
1 / T_{1}=\frac{1}{n} \sum_{i=1}^{n} 1 / T_{1, i},
$$

where $n$ is the number of spins in the group and $T_{1, i}$ is the value of $T_{1}$ attached to the $i$-th spin. A similar expression would hold for $T_{2}$. Equally, one can calculate the average rate for 
each interaction type. For example, one can calculate the average bulk-bulk relaxation rate as well as the bulk-surface relaxation rate, where the latter notation means the $T_{1}$ of a spin (starting) in the bulk interacts with spins on the surface. It is then possible to add the two rates to calculate the overall rate according to

$$
\left(\frac{1}{T_{1}}\right)_{\text {Bulk }}=\left(\frac{1}{T_{1}}\right)_{\text {Bulk-Bulk }}+\left(\frac{1}{T_{1}}\right)_{\text {Bulk-Surface }} .
$$

If there are two distinct classes of spins, then strictly speaking there are two relaxation rates. In the slow exchange limit these show up as two different values of $T_{1}$ in experiments. However, if there is a fast exchange of spins between these two classes, then an average spectrum weighted by the relative populations is observed in experiments ${ }^{1,29}$. In systems of fast exchange a mono-exponential decay of the transverse nuclear magnetisation $\left(T_{2}\right)$ and recovery of equilibrium nuclear magnetisation $\left(T_{1}\right)$ is observed. When exchange is slow, multimodal decay curves are seen, where the number of modes correspond to the number of separate magnetic environments in the system.

\section{Computational details}

A crystal of $11 \AA$ anomalous tobermorite ${ }^{25-27}$, which has the chemical formula

$\mathrm{Ca}_{4} \mathrm{Si}_{6} \mathrm{O}_{15}(\mathrm{OH})_{2} \cdot 5 \mathrm{H}_{2} \mathrm{O}$, was set up such that the plane of the tobermorite sheets defines the $x-y$ plane, measuring $4.6 \times 4.5 \mathrm{~nm}$ in the present case (Figure 1 ). The system consisted of four sheets of $11 \AA$ anomalous tobermorite, except that one of the slit pores was widened to $\sim 1.0 \mathrm{~nm}$, which we shall refer to as the 'gel pore'. This configuration leads to a total of 768 $\mathrm{Ca}$ and $1152 \mathrm{Si}$ atoms in the system. Hence, as per the chemical formula, there were 240 water molecules in each of the three inter-sheet slit pores. The gel pore contained 792 water molecules, making a total of 1512 water molecules in the system. In this model, the oxygen atoms in the silica tertahedra that are at the interface with water are each capped with a hydrogen atom in order to form hydroxyl groups on the surfaces. There were thus a total of 480 hydroxyls in the system.

Molecular dynamics simulations of this system were performed using the DL_POLY package $^{30,31}$ with periodic boundary conditions applied in all three direction. Water molecules in both, slit pores and the gel pore, thus experience confinement effect in the $z$-direction, while 
the $x-y$ plane is effectively infinite due to the periodic boundary condition. The SPC/E potential $^{32}$ was used for the water-water interactions, while the CLAYFF potential ${ }^{33}$ was applied for interactions within the tobermorite crystal. To model the tobermorite-water interface, the Lorentz-Berthelot mixing rules $^{34}$ were applied.

The system was equilibrated using isothermal-isobaric ensemble to a target temperature

of $300 \mathrm{~K}$ and a pressure of $0 \mathrm{~Pa}$. A cut-off distance of $10.0 \AA$ was applied to the potentials and the Ewald summation method ${ }^{35}$ was used to incorporate long-range Coulombic interactions. When equilibrated, the overall dimensions of the entire simulation cell were $4.6 \times 4.5 \mathrm{~nm} \times$ $5.9 \mathrm{~nm}$, which was then simulated using NVT ensemble applying the Hoover thermostat ${ }^{36}$ to obtain a trajectory of $3 \mathrm{~ns}$ for the production of the final results.

\section{RESULTS}

The first task is to identify the distinct classes of nuclear spins in the system. The hydrogen nuclei can be divided into four environments: (1) water in the 'bulk' phase of the gel pore, (2) water adsorbed onto the surfaces of the gel pore, (3) the hydroxyl group hydrogens, which remain attached to the tobermorite crystal and perform dangling motion and (4) water confined in the slit pore regions.

Figure 2 shows the density profile around the gel pore and one slit pore, along the $z$-axis, which is perpendicular to the crystal plane. The other two slit pores, which have very similar density profiles, have been omitted. Peaks in water density near the crystal surface indicate structured water layer formation near the surface. The first major peak in the density of water oxygens on either side of the gel pore can be used to define the water planes. Using this definition we calculated the water residence time on the gel pore surface by plotting the average number of original water oxygens left in the surface layer as a function of time. The average was obtained over multiple time origins. This is shown in the inset of Fig. 2. By fitting an exponential decay function

$$
\langle N(t)\rangle=\langle N(0)\rangle \exp \left(-t / \tau_{S}\right)
$$

where $\langle N(t)\rangle$ is the average number of original water oxygens left on the surface at time $t$, we calculated the water residence time $\tau_{S}$ to be $\sim 30$ ps. Given such a short residence 
time compared to the time taken for a water molecule to diffuse across the pore, this system corresponds to the limit of fast exchange between the surface and the 'bulk' water molecules in the gel pore. Hence, we shall treat all the gel pore water molecules as being equivalent while calculating $T_{1}$.

In addition, given the fact that the hydroxyls remain attached to the surfaces throughout the simulation, the component of the correlation function due to hydroxyl-hydroxyl interactions generally decays negligibly and it is extremely difficult to accurately obtain the Fourier transform of such a flat function. Effectively, this is the rigid lattice limit, where the BPP theory ${ }^{6}$ breaks down. Therefore we do not calculate the $T_{1}$ of the hydroxyls. Specifically, we shall neglect the correlation function of the hydroxyl-hydroxyl and the hydroxyl-water interactions while calculating $T_{1}$ components in the present work. On the other hand, the water-hydroxyl correlation function will be included in the calculation of water $T_{1}$.

Given these assumptions, we are now left with two distinct environments for consideration in the correlation function calculations: water in the entire gel pore and water in the slit pores. Within each of these environments, water molecules perform translational as well as rotational motion. Hence, there are six components of $G^{*}(t)$ arising purely from water in the entire system: translational and rotational components within the gel pore as well as in the slit pores, plus the slit pore-to-gel pore and gel pore-to-slit pore translational correlations. A schematic of how the rotational and translational components are calculated is shown in Fig. 3 , where two water molecules (top and bottom) are shown at $t=0$ (left hand side) and at a later time $t$ (right hand side). The rotational component may be calculated by evaluating the relative position vectors $\mathbf{r}_{0}$ and $\mathbf{r}$ between the two hydrogens in the same water molecule and substituting these in Eq. (4). In this case $\left|\mathbf{r}_{0}\right|=|\mathbf{r}|$ and only the angle between these two vectors changes with time. The translational correlation function is evaluated by considering $\mathbf{r}_{0}$ and $\mathbf{r}$ between hydrogens on separate water molecules.

Figure 4 shows all the six components of $G^{*}(t)$ arising purely due to water, calculated on a per-spin basis. Averaging is performed over multiple time origins as well as over the total number of spins in the given class. A cut-off distance equal to half the simulation box length was applied to construct the neighbour list over which the summation in Eq. (4) is carried out to evaluate the $G^{*}(t)$ contribution due to each spin. This is reasonable in the current 
system, since the $G^{*}(t)$ contributions decay rapidly as the square of the distance ${ }^{10,15}$. For a given spin, the magnitude of $G^{*}(t)$ depends on the number of spins with which it is being correlated, as represented by the summation in Eq. (4). There are different number of spins in the gel pore and the slit pores and hence there is a slight difference in the magnitude of $G^{*}(t)$ of gel pore-slit pore and slit pore-gel pore components. The calculation of the rotational component of $G^{*}(t)$ involves a summation over only one spin, which is the alternate hydrogen on the same water molecule. On the other hand, the translational component of $G^{*}(t)$ is obtained by summing over all the spins on the neighbouring molecules, although their contribution diminishes quickly with increasing distance. These factors are the origin of the different magnitudes of the various components of the correlation function at $t=0$. For water, the closest inter-molecular hydrogen-hydrogen distances are generally larger than the intra-molecular hydrogen-hydrogen distances and since $G^{*}(t)$ scales as the sixth power of these distances at $t=0$, the initial magnitudes of the two rotational components in Fig. 4 are slightly larger than the translational components for their respective environments.

Figure 4 shows that water movement within the slit pores is highly correlated, with the translational component decaying the slowest. This is indicative of highly restricted translational diffusion within the slit pores. The rotational component within the slit pores decays to a slightly larger extent, suggesting that translational movement of these water molecules is more restricted than the rotational movement. The situation is reversed in the gel pore, where the translational component drops more rapidly than the rotational components. Also, the extent of the decay of both these components in the gel pore is much larger than in slit pores. This is due to the larger width available for diffusion in the gel pore, although it can be noted from the rotational component that even in this larger pore width $(\sim 1 \mathrm{~nm})$ water molecules have a tendency to maintain their orientation. The increased noise in the gel pore rotational component is due to poorer statistics coupled with fluctuating movement; there are far fewer intra-molecular spin pairs in the gel pore than intermolecular spin pairs. Finally, the gel pore-slit pore component of the correlation funtion is identical in shape to the slit pore-gel pore component, as expected, but their magnitude is slightly different due to the different number of spins in these two regions. At short times the magnitudes of these two 'cross' correlation components are much smaller than the other 
four components due to the fact that the initial slit pore-gel pore water distances are larger than the nearest water-water distance in either the gel pore or the slit pore. At larger times the cross correlation components appear to be starting to be very similar in magnitude to the translational component in the gel pore.

To underpin these results, mean squared displacements (MSD) ${ }^{37}$ of water in the gel pore and the slit pores were calculated separately. Diffusion in the $x$ - $y$ plane and in the $z$-direction were calculated separately. The MSD is shown as a function of time in Fig. 5. The twodimensional diffusion coefficient, $D_{2 \mathrm{D}}$, was calculated by evaluating the slope of the linear part of the MSD curves through the Einstein equation

$$
D_{2 \mathrm{D}}=\frac{\left\langle(\Delta x)^{2}+(\Delta y)^{2}\right\rangle}{4 \cdot \Delta t},
$$

where $\Delta t=t-t_{0}$ is the time elapsed since the time origin $t_{0}$, while $\Delta x$ and $\Delta y$ are the atomic displacements in the $x$ and $y$ directions respectively since time $t_{0}$. The averaging indicated by the angled brackets was carried out over all the water oxygens in the respective class (gel or slits) as well as over multiple time origins. The two-dimensional diffusion coefficient in the gel pore was thus found to be $1.68 \times 10^{-9} \mathrm{~m}^{2} / \mathrm{s}$. Within the slit pores, this was estimated to be $\sim 9.1 \times 10^{-12} \mathrm{~m}^{2} / \mathrm{s}$, although a trajectory much longer than the $3 \mathrm{~ns}$ reported here would be needed to obtain an accurate value. Classical MD simulations of up to 1 ns of the $9 \AA$ phase of tobermorite ${ }^{38}$ found a value of $5.0 \times 10^{-11} \mathrm{~m}^{2} / \mathrm{s}$ for diffusion in the slit pores ${ }^{39}$. In comparison, ${ }^{1} \mathrm{H}$ NMR field cycling relaxation experiments on cement pastes suggested a value of $2.6 \times 10^{-11} \mathrm{~m}^{2} / \mathrm{s}$ in the slit pores ${ }^{40}$. The experimental value ${ }^{41}$ of bulk water diffusion coefficient is $2.2 \times 10^{-9} \mathrm{~m}^{2} / \mathrm{s}$.

The diffusion coefficient in the gel pore is only $20 \%$ less than the value for bulk water. The reduction in diffusion coefficient is associated with the restricted motion of a fraction of the gel water at the crystal surfaces. On the other hand, the diffusion coefficient in the slit pore is more than two orders of magnitude smaller than that in the gel pore. This explains the slow decay of the $G^{*}(t)$ components compared to those of the gel pore in Fig. 4 and reflects the confinement associated with the crystal interfaces.

The slit pore-gel pore water components of $G^{*}(t)$ presented in Fig. 4 show features appearing at about 1000 ps. We believe that this is due to the water molecules undertaking 
sudden hops to neighbouring sites within the slit pore. This stochastic process cannot be observed directly in a MSD curve due to averaging but is confirmed in Fig. 6, which contains the squared displacements of three randomly-selected water oxygen atoms in the slit pores. There is no averaging over multiple time origins in Fig. 6 and so the hopping motion is revealed by discrete jumps in the coordinate values. These jumps occur on timescales comparable to those where the features are observed in the $G^{*}(t)$ components for the slit pore-gel pore interaction. No features are observed in the slit pore components of $G^{*}(t)$ in Fig. 4 due to the fact that motion within the slit pore is highly correlated and so a hop of a single water molecule makes just a small contribution to the overall $G^{*}(t)$. In order to detect the site jumping process from the correlation function, the motion must be correlated with a different class of molecules. This was additionally confirmed by calculating the correlation of slit pore water spins with a randomly chosen silicon atom of the tobermorite crystal. The $G^{*}(t)$ curve for this interaction also showed similar features to those in the slit pore-gel pore components.

The spin-lattice relaxation times, $T_{1}$, were evaluated using the calculated $G^{*}(t)$ components of Fig. 4 and Eq. (2), as a function of the NMR linear frequency, $f=\omega / 2 \pi$. Contributions arising purely due to water in the gel pore and in the slit pores were calculated separately and are shown in Fig. 7 . The value of $T_{1}$ decreases with frequency in all the components, in agreement with experimental observations ${ }^{40,42,43}$. Also, the translational $T_{1}$ in the slit pores is greater than in the gel pore at high frequencies, but the situation is reversed at lower frequencies. A similar phenomenon is observed for the rotational components and hence also for the overall $T_{1}$ within the two pore types.

As mentioned earlier, since there is no exchange of spins between the gel pore and the slit pores, two separate values of relaxation times would be observed in experiments corresponding to these two distinct regions. Each of these two values may be obtained by adding the relaxation rates arising from the various components within the given region. For instance, the overall value of $T_{1}$ due to purely water in the gel pore, $\left(T_{1}\right)_{\text {Water }}^{\text {Gel }}$, can be evaluated according to

$$
\left(\frac{1}{T_{1}}\right)_{\text {Water }}^{\text {Gel }}=\left(\frac{1}{T_{1}}\right)_{\text {Water, Rot }}^{\text {Gel }}+\left(\frac{1}{T_{1}}\right)_{\text {Water, Trans }}^{\text {Gel }},
$$


where the subscripts Rot and Trans refer to the rotational and translational components respectively. A similar expression can be written for slit pore water. The average $T_{1}$ thus arising from water translation and rotation is also plotted in Fig. 7 for each of these two regions, shown with the diamond and cross symbols.

In addition to the six components of $G^{*}(t)$ purely due to water, there are also additional components arising due to the interaction of hydrogen spins in water with those in the hydroxyl $(\mathrm{OH})$ groups attached on the crystal surfaces. Hydroxyl interactions with gel pore water and slit pore water need to be considered separately. This is because there is no exchange of spins between the gel pore and the slit pores and also because water dynamics in the gel pore is very different from that in the slit pores. Figure 8 shows the components that arise when the hydrogens in the $\mathrm{OH}$ groups are correlated with the hydrogens in water either within the gel pore or within the slit pores. As before, the $G^{*}(t)$ components are averaged over multiple time origins and over the number of water spins in the given region (gel pore or slit pores). In addition, the $\mathrm{OH}-\mathrm{OH}$ component is also shown, which decays negligibly due to the fact that the hydrogen spins in the hydroxyls can only perform a dangling motion and cannot detach from the surface. The hydroxyl-slit water and slit waterhydroxyl components decay more noticeably, but not considerably, due to water motion in the slit pores being highly restricted. Again the magnitudes of these two components are different due to the number of hydroxyl spins and slit pore water spins being different, while their shapes are identical. The hydroxyl-gel water component decays more slowly than the gel pore translational component shown in Fig. 4 initially, but at longer times starts to exhibit similar behaviour to the latter.

The water $T_{1}$ components arising from water-hydroxyl interactions in the gel pore and the slit pores are shown in Fig. 9. The overall water $T_{1}$ within the gel pore was calculated according to

$$
\left(\frac{1}{T_{1}}\right)_{\text {Water }}^{\text {Gel }}=\left(\frac{1}{T_{1}}\right)_{\text {Water-Water }}^{\text {Gel }}+\left(\frac{1}{T_{1}}\right)_{\text {Water-OH }}^{\text {Gel }} .
$$

The overall water $T_{1}$ in the slit pores was calculated separately through a similar expression. These are shown in Fig. 10 as the circle and box symbols. The curves without the hydroxyl contributions, namely the diamond and the cross symbols in Fig. 7 are also reproduced 
in order to illustrate how the presence of hydroxyls lower the value of $T_{1}$ throughout the frequency range. The effect of hydroxyls appears to be smaller in the slit pores compared to the gel pore due to the fact that water motion in the slit pores is much more restricted.

The 3 ns simulations performed here allowed us to obtain $T_{1}$ down to a lower value of $f \sim 400 \mathrm{MHz}$, which just overlaps with frequencies accessible through the current generation of NMR instruments. While the experimentally measured value of bulk water $T_{1}$ at such frequencies is $\sim 3-4 \mathrm{~s}$ at $300 \mathrm{~K}^{44}$, NMR experiments on cement-based materials have measured values of $T_{1}$ that are much smaller than the bulk water value ${ }^{42,43}$. Experimental cement samples invariably contain paramagnetic impurities (e.g. iron) at the surfaces, some of which have much larger values of the gyromagnetic ratio, $\gamma$, which would significantly lower the value of the relaxation time. In our subsequent publications we aim to present calculations including paramagnetic impurities.

At $400 \mathrm{MHz}$, Fig. 10 shows $T_{1}=2.75 \mathrm{~s}$ and $1.47 \mathrm{~s}$ in the gel pore and the slit pores respectively, while the trend in the curve indicates even lower values at lower frequencies. In our earlier work ${ }^{15}$, relaxation time for water confined between two sheets of $\mathrm{SiO}_{2}$, which acted as a gel pore of $\sim 1 \mathrm{~nm}$, was calculated. Also calculated in that study was the relaxation time for bulk water. Curves from that work, obtained by evaluating $T_{1}^{-1}=T_{1 \text {,Rot }}^{-1}+T_{1, \text { Trans }}^{-1}$ for the respective systems, are plotted in Fig. 11. Since the $\mathrm{SiO}_{2}$-water system of that work only contained a gel pore and did not include the hydroxyl contribution, for comparison we reproduce the overall $T_{1}$ arising from the rotational + translational components in the gel pore of the present work (the curve with diamond symbols in Fig. 7) as well as that in the slit pores (cross symbols in Fig. 7). The surface residence time in the $\mathrm{SiO}_{2}$ gel pore was calculated to be 113 ps, which is larger than the 30 ps calculated for the present tobermorite gel pore, suggesting stronger surface-gel water interaction in the $\mathrm{SiO}_{2}$ system. Hence, in Fig. 11 we see a clear trend whereby at lower NMR frequencies, systems with increasingly stronger fluid-surface interactions render decreasing relaxation times. While the tobermorite gel pore surface does not interact as strongly with water as the $\mathrm{SiO}_{2}$ surface did, the tobermorite slit pore surfaces interact much more strongly with water than the $\mathrm{SiO}_{2}$ surface. These results are in qualitative agreement with experimental observations that surface interactions help lower the relaxation time ${ }^{3,42,45}$. 


\section{CONCLUSIONS}

Molecular dynamics simulations of water diffusion in anomalous $11 \AA$ tobermorite, comprising three slit pores and one gel pore, have been performed in order to calculate dipolar correlation functions to yield NMR relaxation times as a function of the Larmor frequency. The components of the correlation function corresponding to different types of environments and motions within the system were calculated separately. The relaxation time of the surface hydroxyls was not calculated in the present study.

The surface residence time of water in the gel pore was very short ( $\sim 30 \mathrm{ps})$, allowing us to neglect the need to treat the surface water differently from that in the bulk phase. While the two-dimensional diffusion coefficient in the gel pore was of the same order of magnitude as in bulk water, it was more than two order of magnitudes smaller in the slit pores. Diffusion in slit pores occurs largely through discreet site hopping.

The motion in the slit pores was found to be much more strongly correlated than in the gel pore owing to the highly restricted nature of motion in the slit pores. This leads to a significant lowering of the relaxation time exclusively within the slit pores. In general, it was found that stronger surface interaction leads to lower relaxation time, in qualitative agreement with experiments. In addition, interaction of water with hydroxyls leads to further lowering of the relaxation times. While interpreting experimental data, it is often helpful to understand how much contribution surface hydroxyls are making to an observed water

relaxation time. This study helps quantify this effect in both the slit pore and the gel pore environments. It was observed that the hydroxyls lower the relaxation time more in the gel pore than in the slit pores.

This study also allows us to quantify the comparative contribution due to rotational and translational motion in the gel pore and slit pore environments. The $T_{1}$ due to rotational motion was found to be lower than that due to translational motion in both the environments at all frequencies. While the difference between rotational and translational $T_{1}$ was found to be a weak function of the Larmor frequency in the gel pore, it was a strong function of the frequency in the slit pores.

The relatively short surface residence time in the gel pore corresponds to the fast exchange 
limit. Our future work will aim to study systems exhibiting slower exchange of surface and bulk phase water. The comparison of the two cases will allow us to better understand the processes underlying experimental data.

\section{ACKNOWLEDGMENTS}

We thank Sandra Galmarini from the EPFL, Switzerland, for useful discussions. N.C.H. and J.S.B. acknowledge the Engineering and Physical Sciences Research Council (UK) for financial support (Grant No. EP/H033343/1). 


\section{References}

1. P. T. Callaghan, Translational Dynamics and Magnetic Resonance (Oxford University Press, 2011).

2. S. Stapf and S.-I. Han, NMR Imaging in Chemical Engineering (Wiley-VCH, 2006).

3. L. Monteilhet, J.-P. Korb, J. Mitchell, and P. J. McDonald, Phys. Rev. E 74, 061404 (2006).

4. R. Valiullin, R. Kimmich, and N. Fatkullin, Phys. Rev. E 56, 4371 (1997).

5. R. Kimmich, Chem. Phys. 284, 253 (2002).

6. N. Bloembergen, E. M. Purcell, and R. V. Pound, Phys. Rev. 73, 679 (1948).

7. D. A. Faux, D. K. Ross, and C. A. Sholl, J. Phys. C: Solid State Phys. 19, 4115 (1986).

8. C. A. Sholl, J. Phys. C: Solid State Phys. 7, 3378 (1974).

9. C. A. Sholl, J. Phys. C: Solid State Phys. 14, 447 (1981).

10. J.-P. Grivet, J. Chem. Phys. 123, 034503 (2005).

11. J. J. Prompers and R. Brüschweiler, J. Am. Chem. Soc. 124, 4522 (2002).

12. K. Åman, E. Lindahl, O. Edholm, P. Håkansson, and P.-O. Westlund, Biophys. J. 84, $102(2003)$.

13. A. Vogel, K.-T. Tan, H. Waldmann, S. E. Feller, M. F. Brown, and D. Huster, Biophys. J. 93, 2697 (2007).

14. A. Villa and G. Stock, J. Chem. Theory Comput. 2, 1228 (2006).

15. D. A. Faux, P. J. McDonald, N. C. Howlett, J. S. Bhatt, and S. V. Churakov, Phys. Rev. E 87, 062309 (2013).

16. A. J. Allen, J. J. Thomas, and H. M. Jennings, Nature Mater. 6, 311 (2007). 
17. E. Masoero, E. Del Gado, R. J.-M. Pellenq, S. Yip, and F.-J. Ulm, Soft Matter 10, 491 (2014).

18. A. Valori, P. J. McDonald, and K. L. Scrivener, Cement Concrete Res. 49, 65 (2013).

19. S. M. Auerbach, K. A. Carrado, and P. K. Dutta, Handbook of Zeolite Science and Technology (CRC Press, 1983).

20. C. Baerlocher, F. Gramm, L. Massüger, L. B. McCusker, Z. He, S. Hovmöller, and X. Zou, Science 315, 1113 (2007).

21. P. Nalawade, B. Aware, V. J. Kadam, and R. S. Hirleka, J. Sci. Ind. Res. 68, 267 (2009).

22. I. G. Richardson, Cement Concrete Res. 34, 1733 (2004).

23. R. F. Feldman and P. J. Sereda, Engineering Journal 53, 53 (1970).

24. H. M. Jennings, Cement Concrete Res. 38, 275 (2008).

25. S. Merlino, E. Bonaccorsi, and T. Armbruster, Eur. J. Mineral. 13, 577 (2001).

26. S. V. Churakov, Eur. J. Mineral. 21, 261 (2008).

27. S. V. Churakov, Am. Mineral. 94, 156 (2009).

28. A. Abragam, The Principles of Nuclear Magnetism (Oxford University Press, 1961).

29. J. R. Zimmerman and W. E. Brittin, J. Phys. Chem. 61, 1328 (1957).

30. W. Smith and T. Forester, J. Molec. Graphics 14, 136 (1996).

31. I. T. Todorov, W. Smith, K. Trachenko, and M. T. Dove, J. Mater. Chem. 16, 1911 (2006).

32. H. J. C. Berendsen, J. R. Grigera, and T. P. Straatsma, J. Phys. Chem. 91, 6269 (1987).

33. R. T. Cygan, J.-J. Liang, and A. G. Kalinichev, J. Phys. Chem. B 108, 1255 (2004).

34. M. P. Allen and D. J. Tildesley, Computer Simulation of Liquids (Oxford University Press, 1989). 
35. P. Ewald, Ann. Phys. 369, 253 (1921).

36. W. G. Hoover, Phys. Rev. A 31, 1695 (1985).

37. D. Frenkel and B. Smit, Understanding Molecular Simulations: from Algorithms to Applications, 2nd ed. (Academic Press, 2002).

38. S. Merlino, E. Bonaccorsi, and T. Armbruster, Eur. J. Mineral. 12, 411 (2000).

39. A. G. Kalinichev, J. Wang, and R. J. Kirkpatrick, Cement Concrete Res. 37, 337 (2007).

40. J.-P. Korb, P. J. McDonald, L. Monteilhet, A. G. Kalinichev, and R. J. Kirkpatrick, Cement Concrete Res. 37, 348 (2007).

41. K. Krynicki, C. D. Green, and D. W. Salter, Faraday Discuss. Chem. Soc. 66, 199 (1978).

42. S. Godefroy, J.-P. Korb, M. Fleury, and R. G. Bryant, Phys. Rev. E 64, 021605 (2001).

43. F. Barberon, J.-P. Korb, D. Petit, V. Morin, and E. Bermejo, Phys. Rev. Lett. 90, $116103(2003)$.

44. J. C. Hindman, A. Svirmickas, and M. Wood, J. Chem. Phys. 59, 1517 (1973).

45. P. J. McDonald, J.-P. Korb, J. Mitchell, and L. Monteilhet, Phys. Rev. E 72, 011409 (2005). 
Figure 1: Snapshot of the simulation setup. The larger (cyan) spheres are Ca atoms. Si chains are shown by yellow polyhedra. Water molecules are shown by two-colour thin sticks. Surface OH groups are the smaller (red and white) spheres.

Figure 2: The axial density profile of the chemical species around the gel pore and an adjacent slit pore. $\mathrm{O}_{\mathrm{w}}$ and $\mathrm{H}_{\mathrm{w}}$ are water oxygen and hydrogen respectively, while $\mathrm{Si}$ and $\mathrm{O}_{\mathrm{SiO}}$ denote silicon and oxygen atoms in the $\mathrm{SiO}_{2}$ tetrahedra. Calcium and hydroxyls are omitted in this figure for clarity. The first significant peak in water oxygen on either side of the gel pore region is used to define a surface layer of water. The inset shows the time-decay of the number of water molecules that start on the gel pore surface at $t=0$, averaged over multiple time origins.

Figure 3: An illustration of the manner in which rotational and translational components of $G^{*}(t)$ for water are calculated. In Eq. (4), calculating $\mathbf{r}_{0}$ and $\mathbf{r}$ between hydrogen atoms in the same water molecule gives the rotational component. To calculate the translational component of the correlation function, these vectors are evaluated between two hydrogen atoms on separate water molecules.

Figure 4: The time-evolution of the inter-water and intra-water components of the powderaveraged dipolar correlation function. The top two curves, marked with the occasional box and cross symbols, arise from the translation and rotation of water molecules within the slit pores. The middle two curves, marked with the occasional triangle and diamond symbols, arise from translation and rotation of water molecules in the gel pore. The bottom two curves show the cross-correlation between the gel pore and the slit pore water, marked woth the plus and circle symbols. 
Figure 5: Mean squared displacement of water oxygens in the three slit pores (top) and the gel pore (bottom). The squares show the two-dimensional component of the diffusion in the $x-y$ plane, namely $\left\langle(\Delta x)^{2}+(\Delta y)^{2}\right\rangle$, while the circles show the mean squared displacement in the $z$-direction, $\left\langle(\Delta z)^{2}\right\rangle$. For visual clarity, the square and circle symbols are only exemplar and do not mark every calculated data point.

Figure 6: Single time origin displacement curves for some randomly chosen atoms in the slit pores. The squares show the squared displacement in the $x$-y plane, namely $(\Delta x)^{2}+(\Delta y)^{2}$ with respect to the time origin, while the circles show the squared displacement in the $z$-direction, $(\Delta z)^{2}$. For visual clarity, the square and circle symbols do not mark every calculated data point.

Figure 7: Components of the NMR relaxation time of water, purely due to rotational and translational movement in the gel pore and the slit pores, as a function of the NMR linear frequency. The water-hydroxyl interaction is not included.

Figure 8: The time-evolution of the powder-averaged components of the dipolar correlation function arising from hydroxyl interactions. The $\mathrm{OH}-$ gel water and gel water-OH components, marked with the occasional cross and triangle symbols, decay much faster than the $\mathrm{OH}-$ slit water and slit water-OH components, marked with the occasional circles and boxes. The $\mathrm{OH}-\mathrm{OH}$ component decays negligibly.

Figure 9: Components of the NMR relaxation time of water, purely due to water-hydroxyl interactions within the gel pore and the slit pores, as a function of the NMR linear frequency. The water-water interactions are not included.

Figure 10: NMR relaxation time of water as a function of the linear frequency within the gel pore and the slit pores, with and without hydroxyl interactions included. 
Figure 11: The overall NMR relaxation time for the entire system as a function of the NMR linear frequency, along with the results of earlier work of Faux et al. ${ }^{15}$. Circles and squares show the results of bulk water and an $\mathrm{SiO}_{2}$ gel pore respectively from the earlier study. 


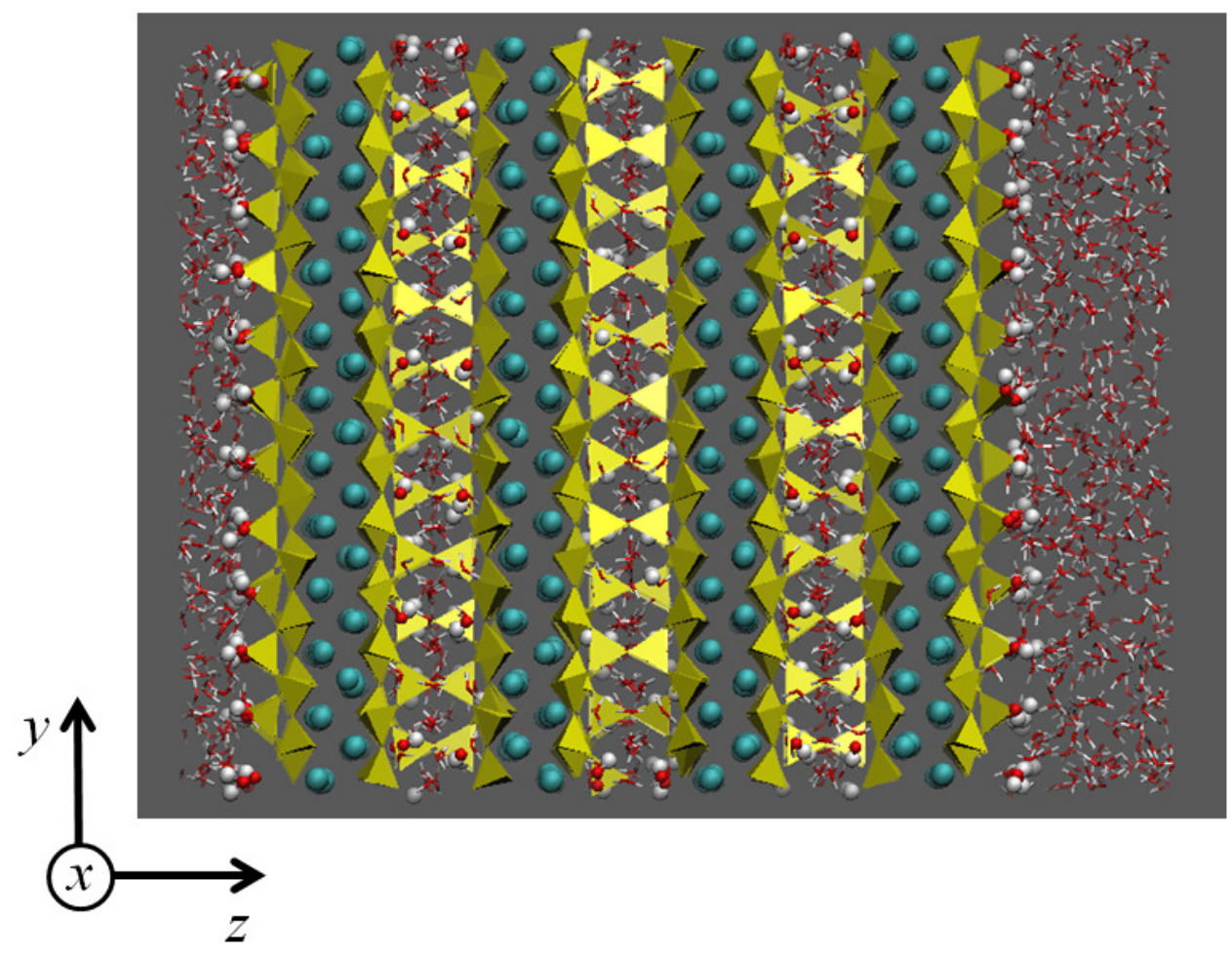

Figure 1

Jayesh S. Bhatt, Peter J. McDonald, David A. Faux, Nicholas C. Howlett, Sergey V. Churakov Int. J. Quant. Chem. 


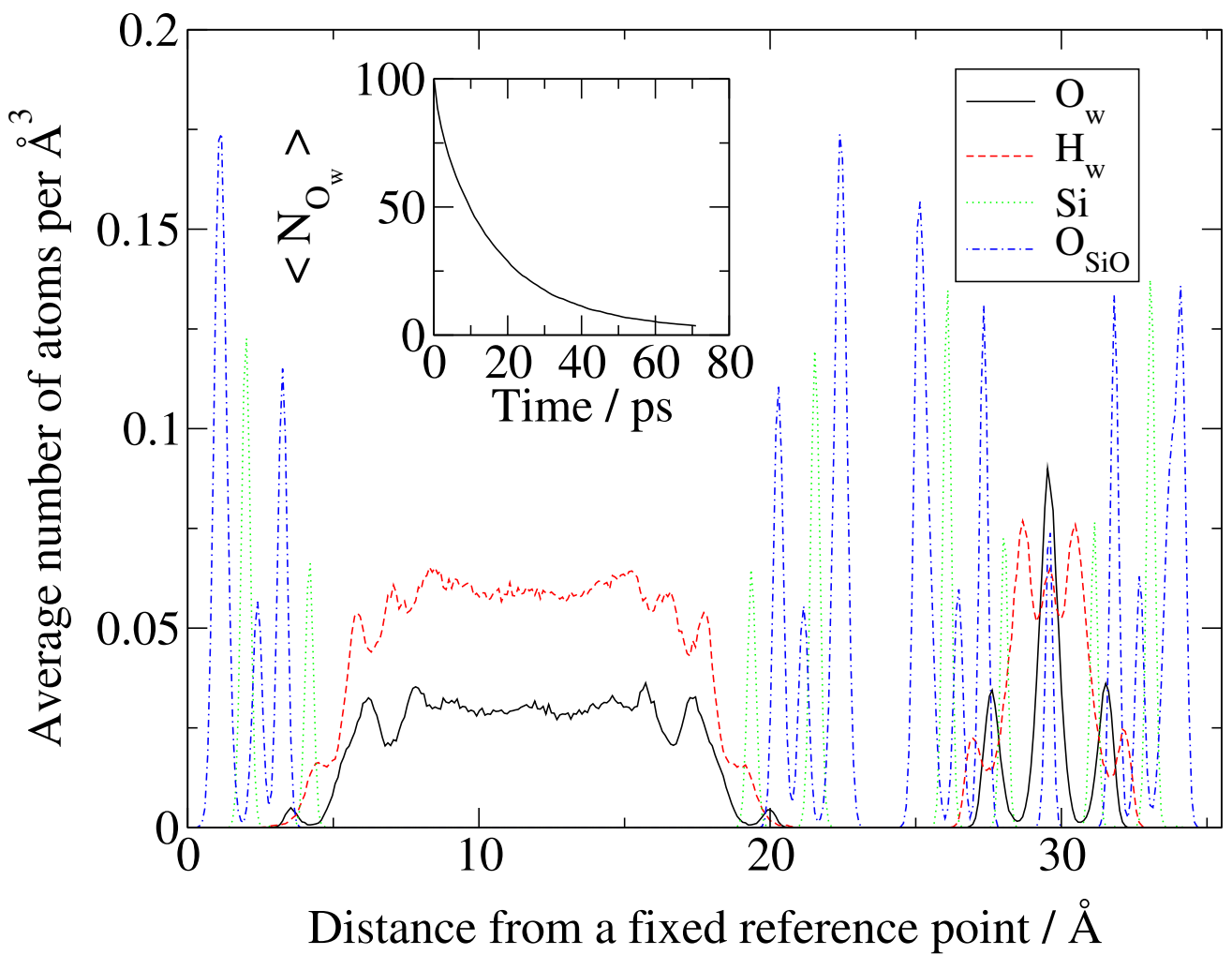

Figure 2

Jayesh S. Bhatt, Peter J. McDonald, David A. Faux, Nicholas C. Howlett, Sergey V.

Churakov Int. J. Quant. Chem. 


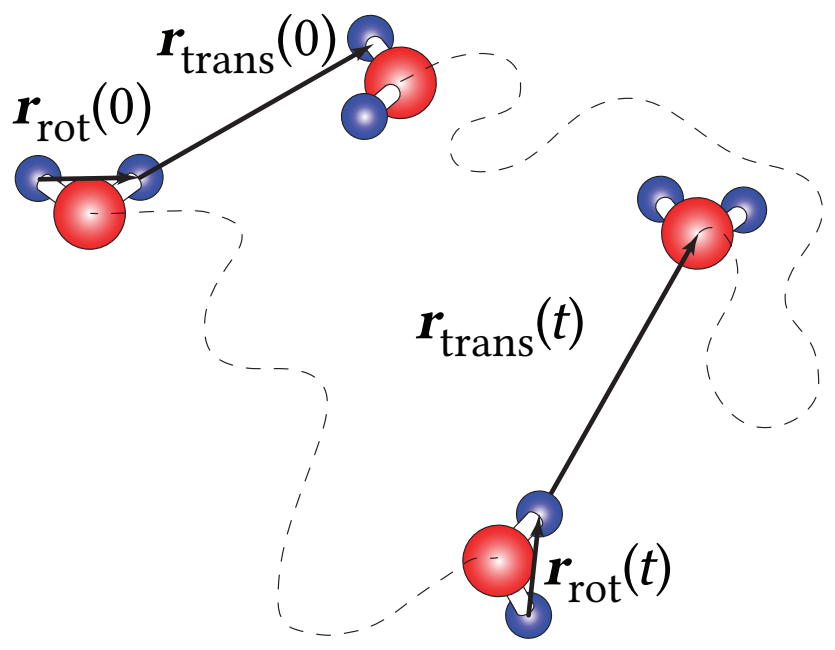

Figure 3

Jayesh S. Bhatt, Peter J. McDonald, David A. Faux, Nicholas C. Howlett, Sergey V. Churakov Int. J. Quant. Chem. 


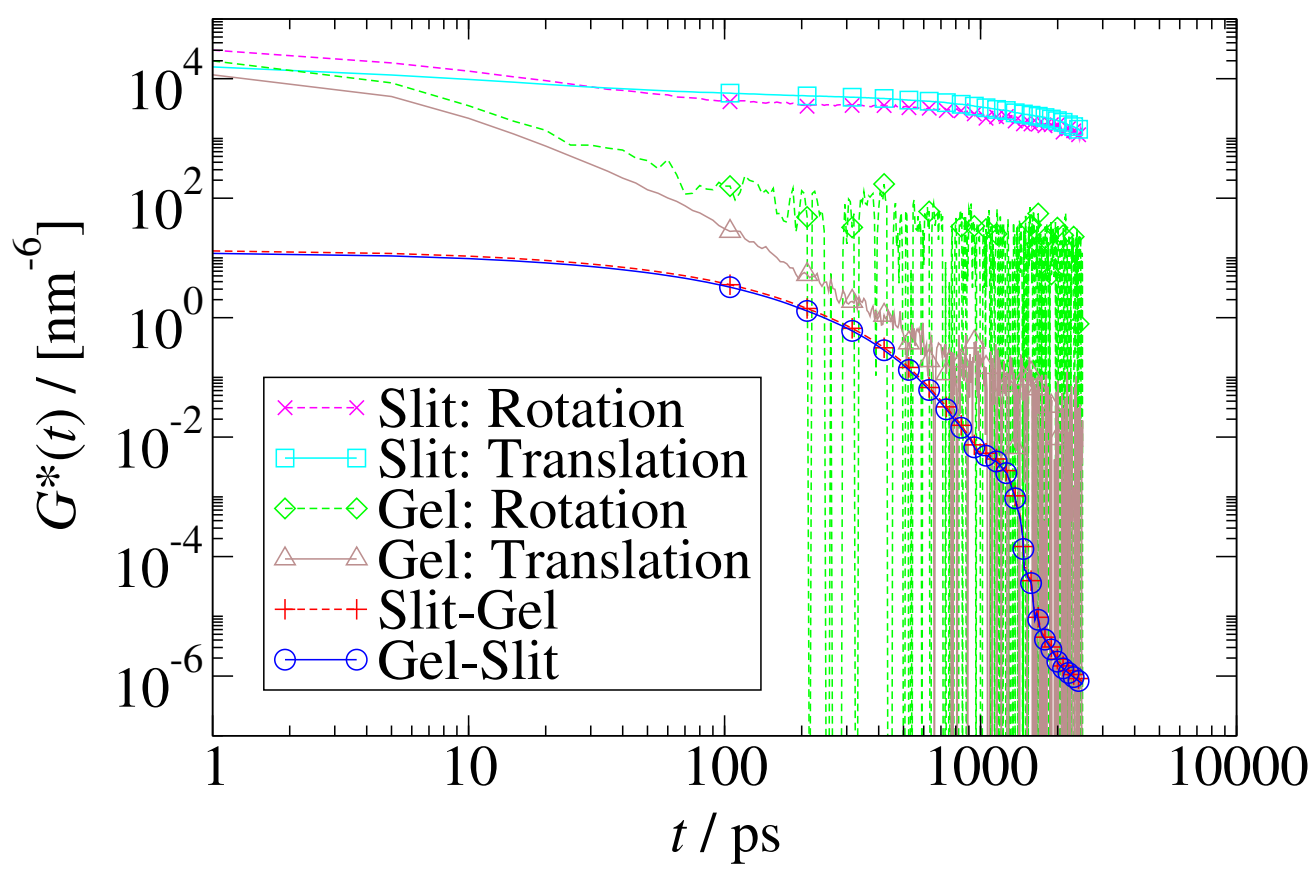

Figure 4

Jayesh S. Bhatt, Peter J. McDonald, David A. Faux, Nicholas C. Howlett, Sergey V.

Churakov

Int. J. Quant. Chem. 


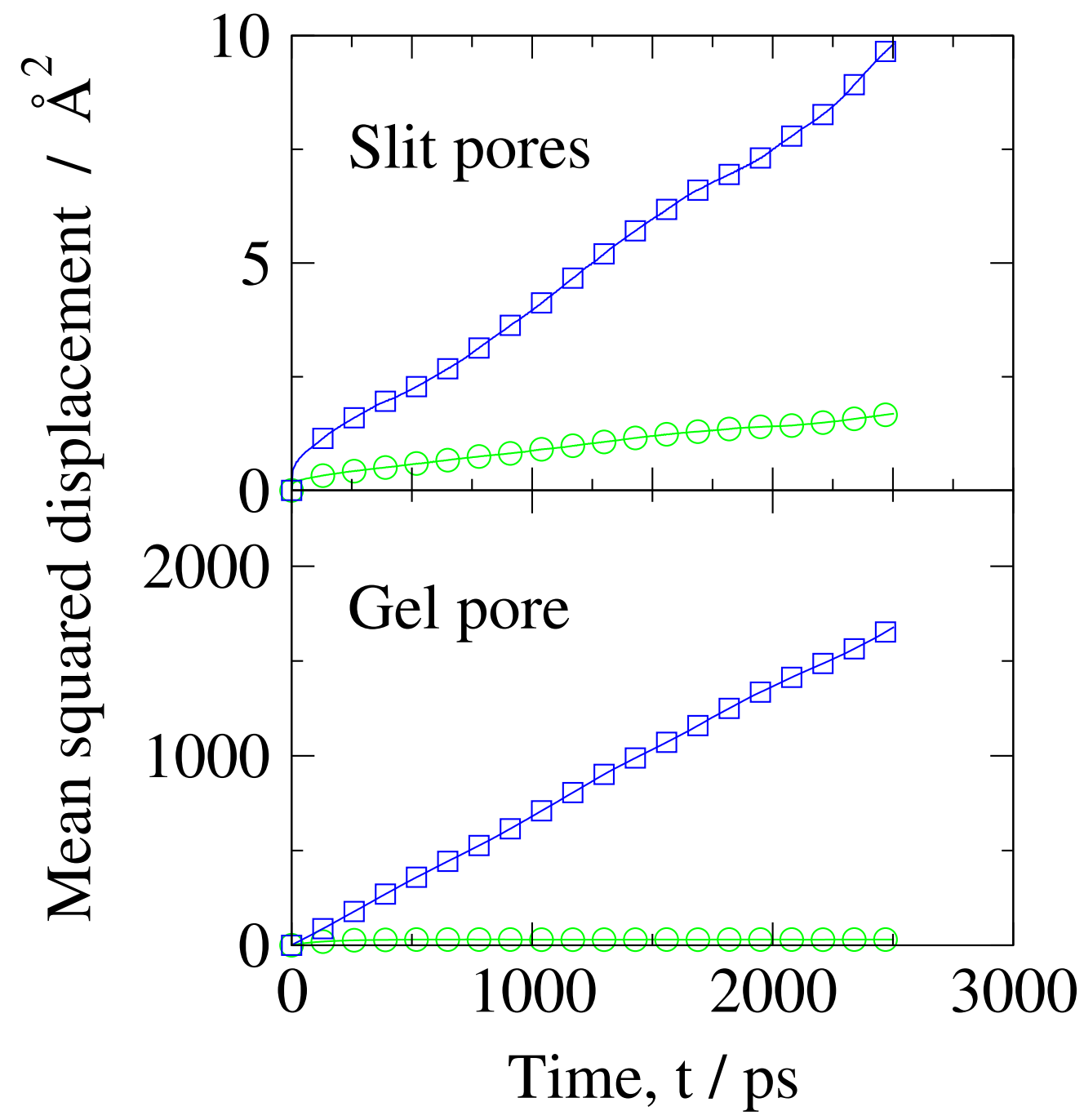

Figure 5

Jayesh S. Bhatt, Peter J. McDonald, David A. Faux, Nicholas C. Howlett, Sergey V. Churakov Int. J. Quant. Chem. 


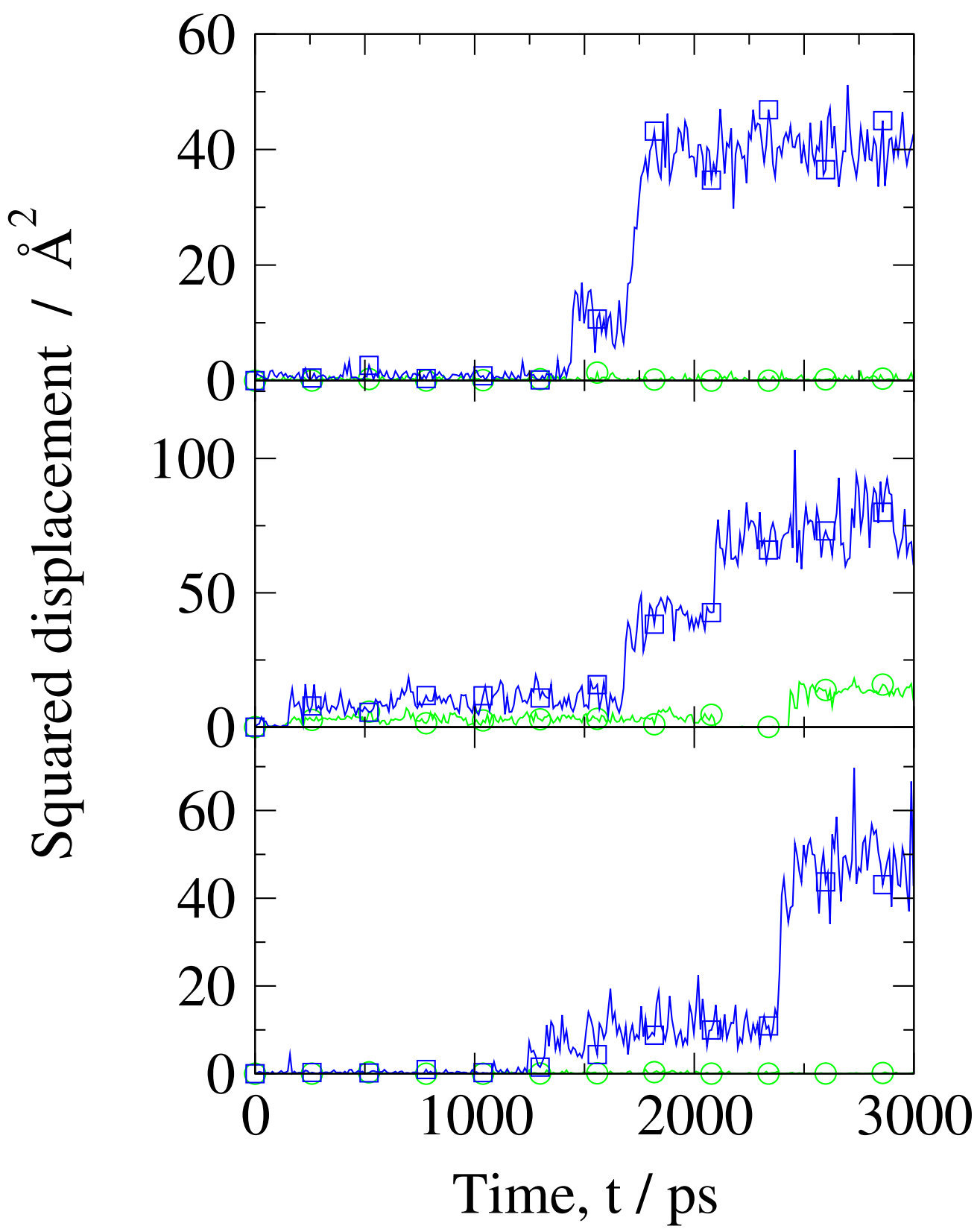

Figure 6

Jayesh S. Bhatt, Peter J. McDonald, David A. Faux, Nicholas C. Howlett, Sergey V. Churakov Int. J. Quant. Chem. 


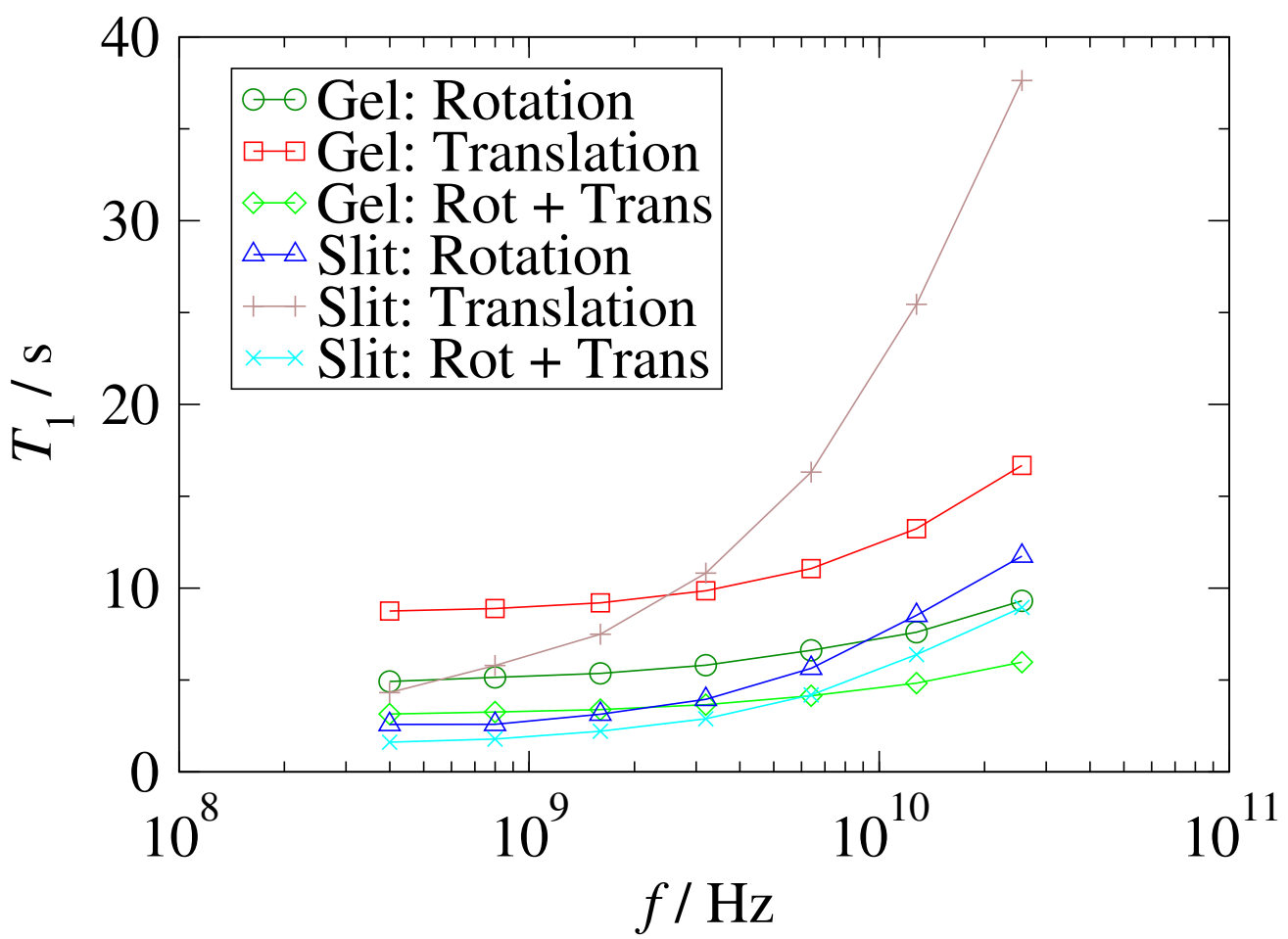

Figure 7

Jayesh S. Bhatt, Peter J. McDonald, David A. Faux, Nicholas C. Howlett, Sergey V.

Churakov Int. J. Quant. Chem. 


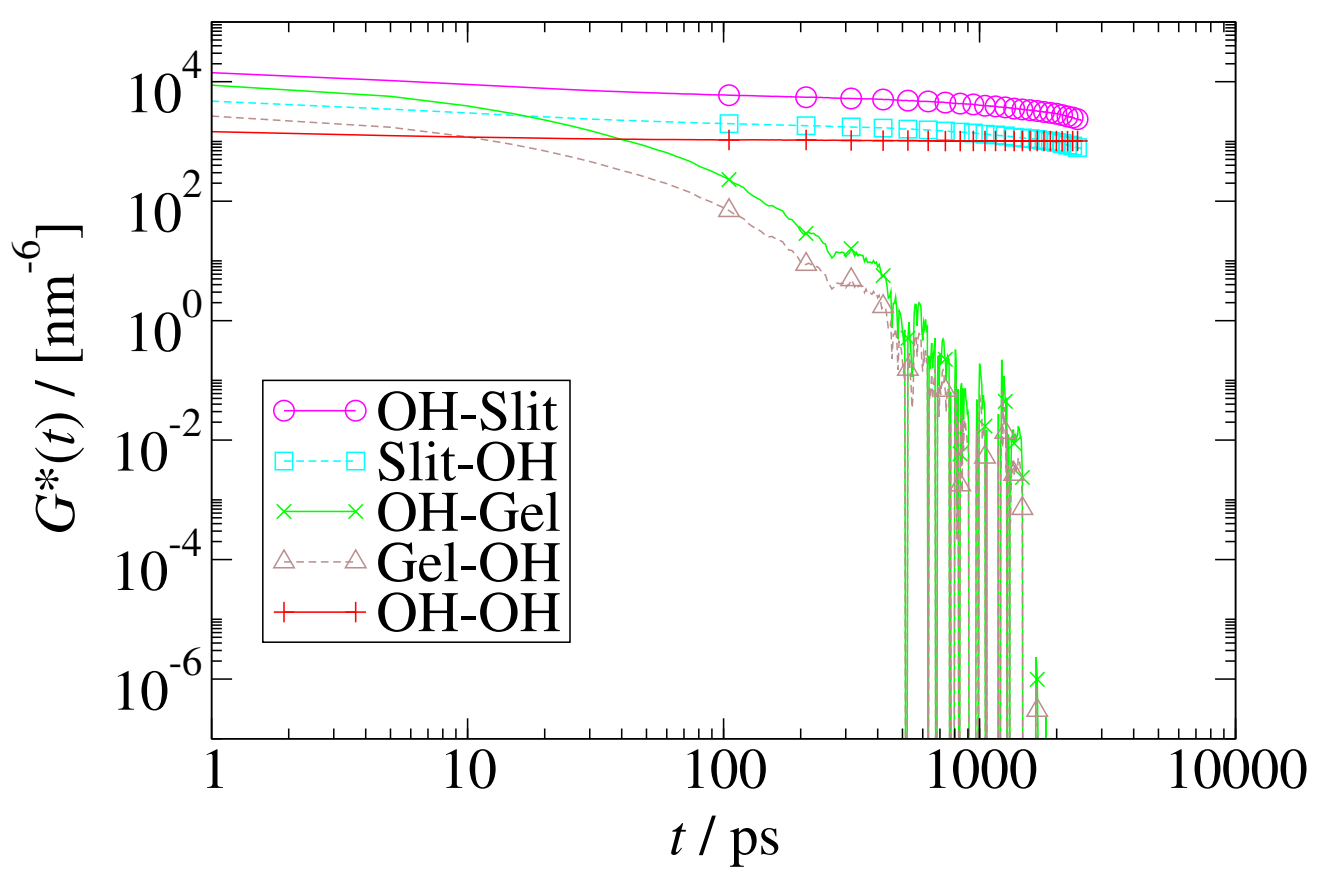

Figure 8

Jayesh S. Bhatt, Peter J. McDonald, David A. Faux, Nicholas C. Howlett, Sergey V. Churakov Int. J. Quant. Chem. 


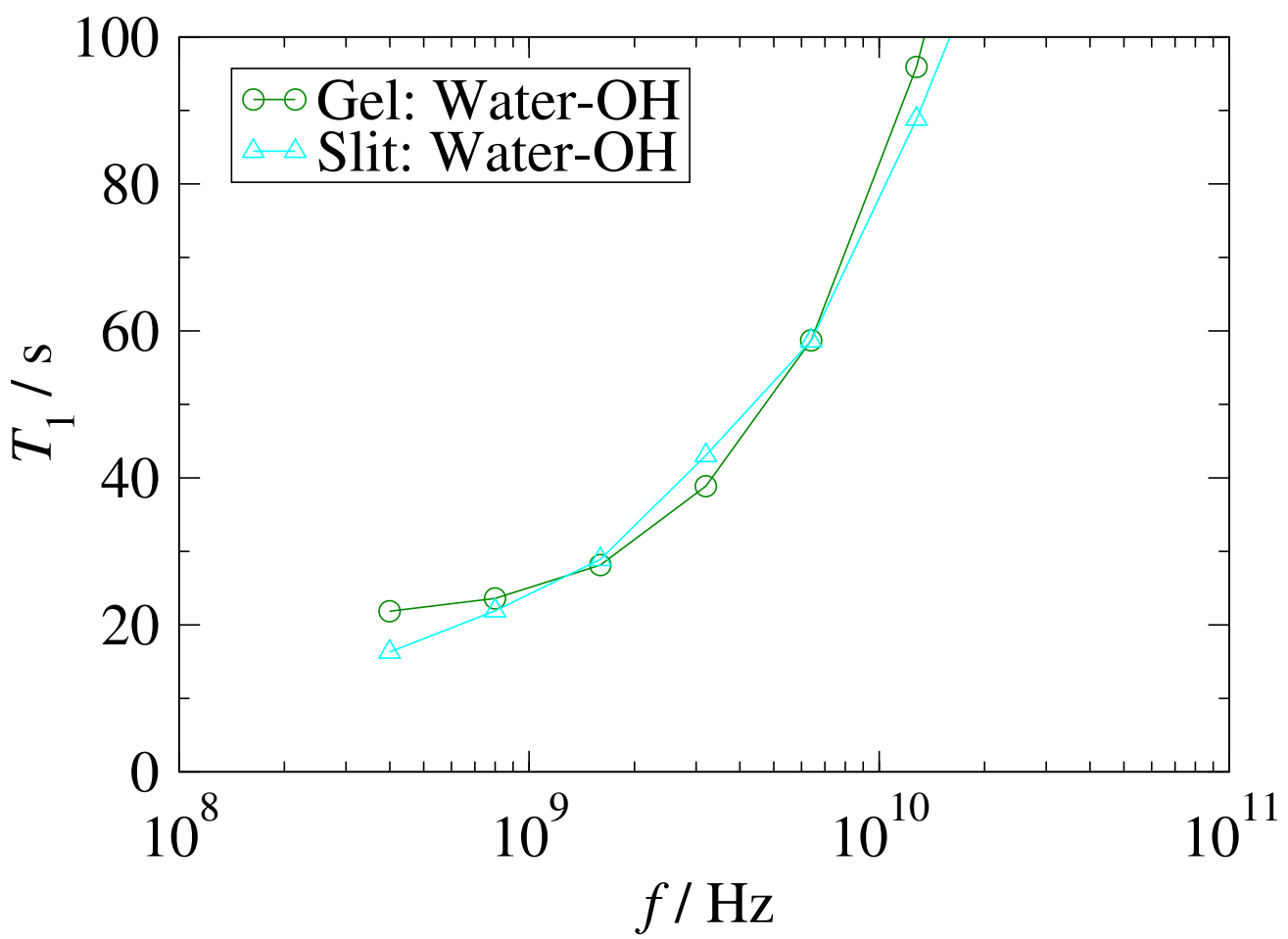

Figure 9

Jayesh S. Bhatt, Peter J. McDonald, David A. Faux, Nicholas C. Howlett, Sergey V.

Churakov Int. J. Quant. Chem. 


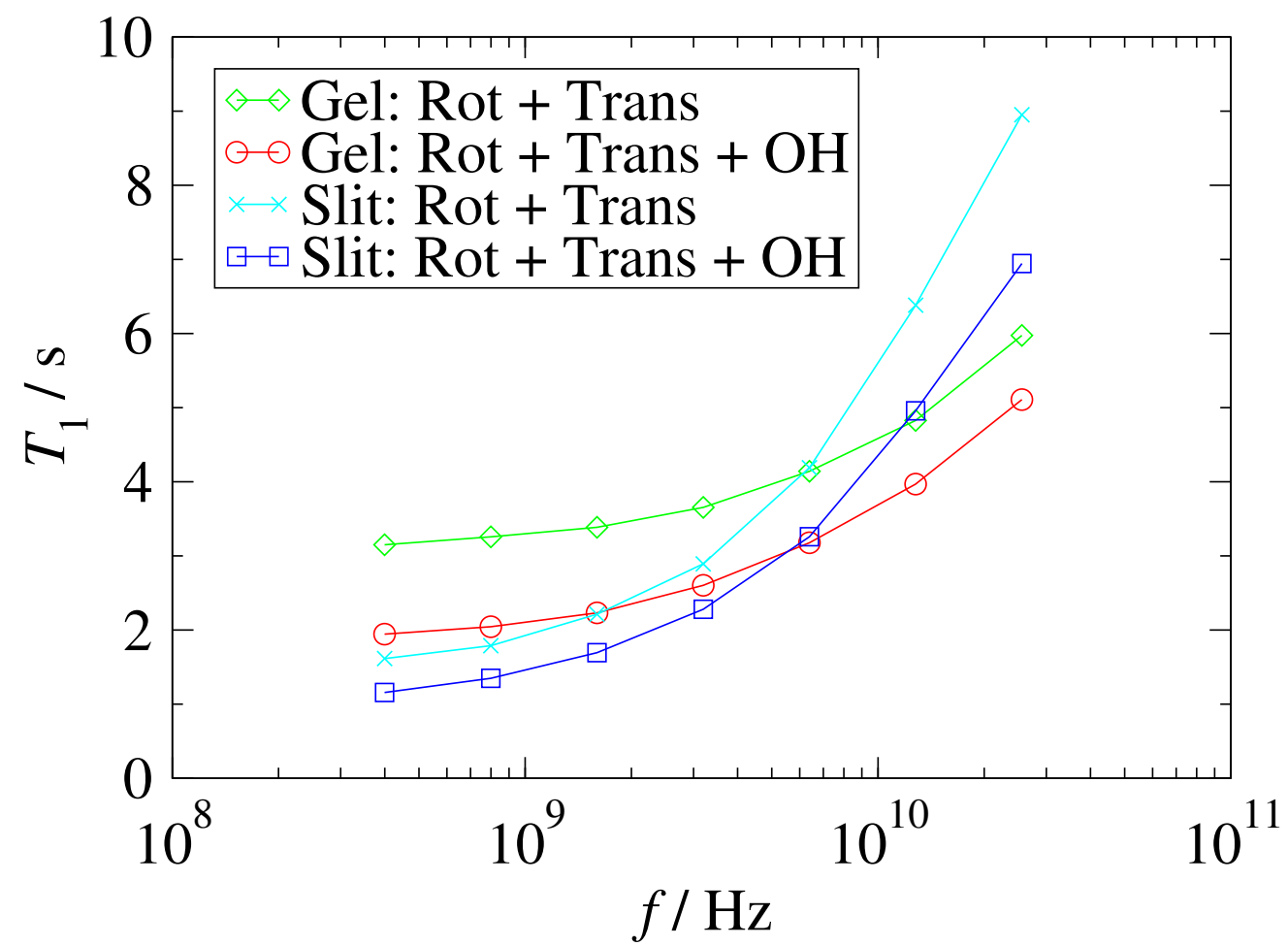

Figure 10

Jayesh S. Bhatt, Peter J. McDonald, David A. Faux, Nicholas C. Howlett, Sergey V. Churakov Int. J. Quant. Chem. 


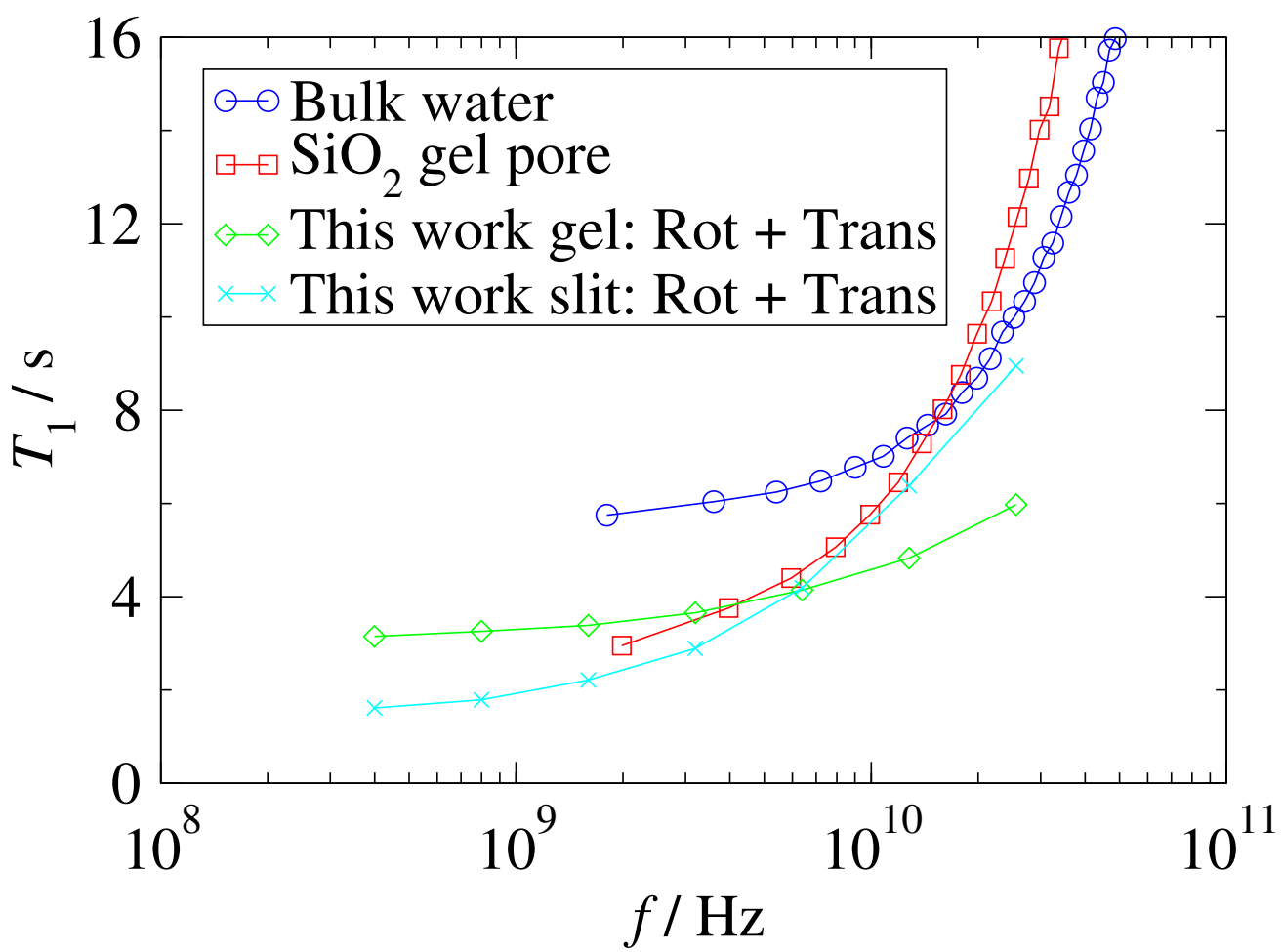

Figure 11

Jayesh S. Bhatt, Peter J. McDonald, David A. Faux, Nicholas C. Howlett, Sergey V. Churakov Int. J. Quant. Chem. 